Study design A qualitative semi-structured interview format was utilised. Purposive sampling was used to recruit 10 parents who either consented or declined autopsy from a large hospital, where there were 30 stillbirths in 2011. Interpretative phenomenological analysis (IPA) was employed as the analytic strategy. IPA allows for close examination of parents' experiences using a small purposive sample by identifying superordinate themes which highlight what is important to the participant but also detail the meaning of these phenomena in a social context.

Results Findings revealed four superordinate themes influencing parents' decision-making; attribution of death, searching for meaning, knowledge of the autopsy procedure and protective parent. Parents discussed the need for the certainty of the diagnosis as it influenced emotional reactions including difficulty in coping with the uncertainty of the outcome of a future pregnancy. Parents, who declined autopsy, strongly indicated that the key reason was to protect their child from further harm. Parents' knowledge and understanding of the autopsy process was acquired primarily from public discourse, with particular reference to television programmes, which elicited negative responses from parents due to their perception of the invasive nature of the autopsy process.

Conclusion These findings have implications for psychological models of decision making and clinical practise. This study underscores the challenges that clinicians face in overcoming public misperceptions of the invasiveness of some autopsy procedures.

\section{PP.47 EVALUATING OUTCOMES OF SKELETAL DYSPLASIAS (SDS)}

doi:10.1136/archdischild-2013-303966.327

PD Perry, JLS Budd, ES Draper. East Midlands \& South Yorkshire Congenital Anomaly Register, Dept. Health Sciences, University of Leicester, Leicester, UK

Objective To evaluate the outcome of 543 pregnancies identified with a skeletal dysplasia or an antenatal suspected skeletal anomaly in the East Midlands and South Yorkshire (EMSYCAR) Congenital Anomaly Register over a fifteen year period.

Background Skeletal dysplasias form a large group of congenital anomalies affecting cartilage and bone growth. Strongly associated with syndromes and underlying genetic conditions, they vary in severity from lethal achondroplasias to milder osteochondroplasias. The UK Fetal Anomaly Screening Programme antenatal detection target for lethal SDs is $60 \%$. Given the problems with definitive antenatal diagnosis this is difficult to achieve.

Methods Between 1997 and 2011, 982,073 births were monitored by EMSYCAR; 543 cases were identified with a SD or antenatally suspected skeletal anomaly (a birth prevalence of 5.53/10,000 births). Each case was individually reviewed to ascertain the type and severity of the skeletal anomaly as ICD-10 codes alone cannot adequately perform this function.

Results $62(11.4 \%)$ of the 543 had fully resolved by delivery. 77 $(18.4 \%)$ resulted from a chromosomal anomaly, and 65 (12.0\%) had other structural anomalies. The remaining 339 (62.4\%) had a skeletal anomaly (3.45/10,000), of which 206 had a SD. Of these 77 were non-lethal and 129 lethal $(1.31 / 10,000)$. Overall, 93\% of lethal SDs were identified antenatally, $63 \%$ within the FASP screening window $\left(<20^{+6}\right.$ weeks gestation).

Conclusion Although only $38 \%$ of total cases had an isolated actual or suspected skeletal anomaly, almost two-thirds of those were lethal SDs. The vast majority were antenatally diagnosed and the FASP target achieved.

\section{PP.48 THE IMPACT OF SEVERE MATERNAL MORBIDITY ON PSYCHOLOGICAL HEALTH AT 6-8 WEEKS POSTPARTUM - A PROSPECTIVE COHORT STUDY IN ENGLAND}

doi:10.1136/archdischild-2013-303966.328

M Furuta, J Sandall, D Bick. King's College London, London, UK
Background The incidence of severe maternal morbidity (SMM) is increasing in high-income countries as a consequence of higher caesarean section rates and more complex health needs of women who become pregnant. Access to emergency obstetric care means that for the majority of these women, SMM is unlikely to result in loss of life. However, little was known about the impact on postnatal morbidity.

Aim To assess the impact of SMM (defined as major obstetric haemorrhage, severe hypertensive disorders, critical care unit admission) on maternal health, focusing particularly on post-traumatic stress disorder (PTSD) symptoms at 6-8 weeks postpartum.

Method A prospective cohort study was undertaken of women who gave birth over six months in 2010 in one large inner city maternity unit in England. Data on health outcomes were collected on 1824 women using self-administered questionnaires at 6-8 weeks postpartum (response rate $=53 \%$ ). Multivariable logistic regression analysis examined the relationship between SMM and PTSD symptoms taking into account factors that might influence the relationship. Ethics approval was obtained.

Results There was a higher risk of PTSD symptoms following SMM (OR $=3.22,95 \% C I=1.62-6.43, p=0.001)$ after adjusting for all potential confounding factors. Women's higher perceived control during labour and birth and better neonatal outcomes slightly reduced the effect size of SMM on PTSD symptoms.

Conclusion Findings have important implications for women's health, and the content and organisation of maternity services. Women and clinicians should be aware that SMM can trigger symptoms of PTSD, with further work required to promote care to prevent these symptoms.

\section{PP.49 WITHDRAWN BY AUTHOR}

\section{PP.50 WITHDRAWN BY AUTHOR}

\section{PP.51 PERINATAL OUTCOMES IN TWIN PREGNANCY IN IRELAND}

doi:10.1136/archdischild-2013-303966.329

'AM O' Mahony, ${ }^{2} \mathrm{~S}$ Meaney, ${ }^{3} \mathrm{~K}$ O' Donoghue. 'School of Medicine, University College Cork, Ireland; 'National Perinatal Epidemiology Centre, University College Cork, Ireland; ${ }^{3}$ Dept. of Obstetrics and Gynaecology, University College Cork, Ireland

Introduction Over the past two decades multiple births have been increasing in Ireland, in 201117.9 sets of twins per 1,000 live births were born. This study aims to investigate adverse perinatal outcomes in twin pregnancies.

Methods A retrospective cohort study of all twin pregnancies delivered from 2009 to 2011 in a large, tertiary hospital $(\sim 8,000$ deliveries per annum) in the Republic of Ireland was conducted. Birth registers, NCIU and clinic records were reviewed to examine perinatal outcomes.

Results Of the 523 twin pregnancies included in the study mean gestational age at delivery was $35.1 \pm 3.8$ (weeks). $79.1 \%(n=413)$ delivered preterm ( $<37$ weeks) of which $75.8 \%(n=313)$ were classified as late preterm infants, delivering between 34-37 weeks. Among the 523 twins 47.5\% ( $n=247)$ were nulliparous and $16.3 \%$ $(\mathrm{n}=87)$ were monochorionic $(\mathrm{MC})$. Nulliparity and $\mathrm{MC}$ were both significantly associated with preterm delivery $(p=0.02$ and $p<0.001$, respectively). Both had lower mean gestational ages $(p=0.007$ and $p<0.001$, respectively) with significant lower birth weights $(p<0.001)$ compared to parous and dichorionic $(D C)$ pregnancies. Intra-uterine fetal death $(11.9 \%$ vs. $1.3 \% ; p<0.001)$, TTTS $(24.1 \%$ vs. $0.1 \% ; p<0.001)$ and perinatal mortality $(p=0.002)$ were higher in MC pregnancies compared to DC. Mean maternal age was $33.2 \pm 4.9$ years and fetal anomalies increased with advanced maternal age; $<40$ years of age, $(p=0.01)$. 